(C)Timofeev E. A., 2016

DOI: $10.18255 / 1818-1015-2016-2-185-194$

UDC 519.987

\title{
Asymptotic Formula for the Moments of Bernoulli Convolutions
}

\author{
Timofeev E. A.
}

Received February 8, 2016

Abstract.

For each $\lambda, 0<\lambda<1$, we define a random variable

$$
Y_{\lambda}=(1-\lambda) \sum_{n=0}^{\infty} \xi_{n} \lambda^{n}
$$

where $\xi_{n}$ are independent random variables with

$$
\mathrm{P}\left\{\xi_{n}=0\right\}=\mathrm{P}\left\{\xi_{n}=1\right\}=\frac{1}{2} .
$$

The distribution of $Y_{\lambda}$ is called a symmetric Bernoulli convolution. The main result of this paper is

$$
M_{n}=\mathrm{E} Y_{\lambda}^{n}=n^{\log _{\lambda} 2} 2^{\log _{\lambda}(1-\lambda)+0.5 \log _{\lambda} 2-0.5} e^{\tau\left(-\log _{\lambda} n\right)}\left(1+\mathcal{O}\left(n^{-0.99}\right)\right),
$$

where

$$
\tau(x)=\sum_{k \neq 0} \frac{1}{k} \alpha\left(-\frac{k}{\ln \lambda}\right) e^{2 \pi i k x}
$$

is a 1-periodic function,

$$
\alpha(t)=-\frac{1}{2 i \operatorname{sh}\left(\pi^{2} t\right)}(1-\lambda)^{2 \pi i t}\left(1-2^{2 \pi i t}\right) \pi^{-2 \pi i t} 2^{-2 \pi i t} \zeta(2 \pi i t),
$$

and $\zeta(z)$ is the Riemann zeta function.

The article is published in the author's wording.

Keywords: moments, self-similar, Bernoulli convolution, singular, Mellin transform, asymptotic

For citation: Timofeev E. A., "Asymptotic Formula for the Moments of Bernoulli Convolutions", Modeling and Analysis of Information Systems, 23:2 (2016), 185-194.

On the authors:

Timofeev Evgeniy Alexandrovich, orcid.org/0000-0002-0980-2507, ScD, professor

P.G. Demidov Yaroslavl State University,

Sovetskaya str., 14, Yaroslavl, 150000, Russia, e-mail: timofeevEA@gmail.com 
For each $\lambda, 0<\lambda<1$ we define the random variable

$$
Y_{\lambda}=(1-\lambda) \sum_{n=0}^{\infty} \xi_{n} \lambda^{n}
$$

where $\xi_{n}$ are independent random variables with

$$
\mathrm{P}\left\{\xi_{n}=0\right\}=\mathrm{P}\left\{\xi_{n}=1\right\}=\frac{1}{2} .
$$

The distribution of $Y_{\lambda}$ is called a symmetric Bernoulli convolution.

The cumulative distribution function $F_{\lambda}(t)=\mathrm{P}\left\{Y_{\lambda}<t\right\}$ can be characterized by the functional equation

$$
F_{\lambda}(x)=\frac{1}{2} F_{\lambda}\left(\lambda^{-1} x\right)+\frac{1}{2} F_{\lambda}\left(\lambda^{-1} x-\lambda^{-1}+1\right), \quad 0 \leq x \leq 1 .
$$

We stress that the Fourier transform is the infinite product

$$
\phi(t)=\mathrm{E} e^{i t Y_{\lambda}}=\prod_{n=0}^{\infty}\left(\frac{1}{2}+\frac{1}{2} e^{i t \lambda^{n}(1-\lambda)}\right)=e^{i t / 2} \prod_{n=0}^{\infty} \cos \left(\lambda^{n}(1-\lambda) t / 2\right),
$$

The early study of $F_{\lambda}(t)$ was related to some questions of harmonic analysis [1, 14.20].

In Figure 1 we show the histograms for $F_{\lambda}^{\prime}(t)$ approximations. This graphics were created as Iterated Functions System of the maps $S_{1}(x)=\lambda x, S_{2}(x)=\lambda x-\lambda+1$. We used $2^{20}$ points and 1000 equally intervals for the histogram.

We stress that these approximations are very crude.

Since the 1930's a lot of work has been done to investigate $F_{\lambda}(t)$ (see e.g. survey [8]).

One of the fundamental question is to decide for which $\lambda$ the function $F_{\lambda}(t)$ is absolutely continuous and for which it is singular.

Results on absolute continuity of $F_{\lambda}(t)$.

- Jessen and Wintner [7] proved that $F_{\lambda}(t)$ is either absolutely continuous or purely singular. It is clear that $F_{\lambda}(t)$ is uniform on $[0,1]$ for $\lambda=\frac{1}{2}$ and is purely singular for $\lambda<\frac{1}{2}$.

- Wintner [13] proved that $F_{\lambda}(t)$ is absolutely continuous for $\lambda=2^{-1 / k}, k=1,2, \ldots$, with a density having $k-1$ derivatives. For example, for $\lambda=2^{-1 / 2}$ we have the following density

$$
F_{\lambda}^{\prime}(x)= \begin{cases}\left(2+\frac{3}{\sqrt{2}}\right) x, & 0 \leq x \leq \sqrt{2}-1 \\ 1+\frac{1}{\sqrt{2}}, & \sqrt{2}-1 \leq x \leq 2-\sqrt{2} \\ \left(2+\frac{3}{\sqrt{2}}\right)(1-x), & 2-\sqrt{2} \leq x \leq 1 .\end{cases}
$$

- Erdös [4] showed that $F_{\lambda}(t)$ is singular when $\lambda^{-1}$ is a Pisot (Pisot-Vijayaraghavan) number $(0.5<\lambda<1)$. Moreover, the Fourier transform $\phi(t)$ does not tend to 0 as $t \rightarrow \infty$. Recall that a Pisot number is an algebraic integer $\theta>1$ all of whose Galois conjugates (other roots of the minimal polynomial) of $\theta$ are less than 1 in modulus. 

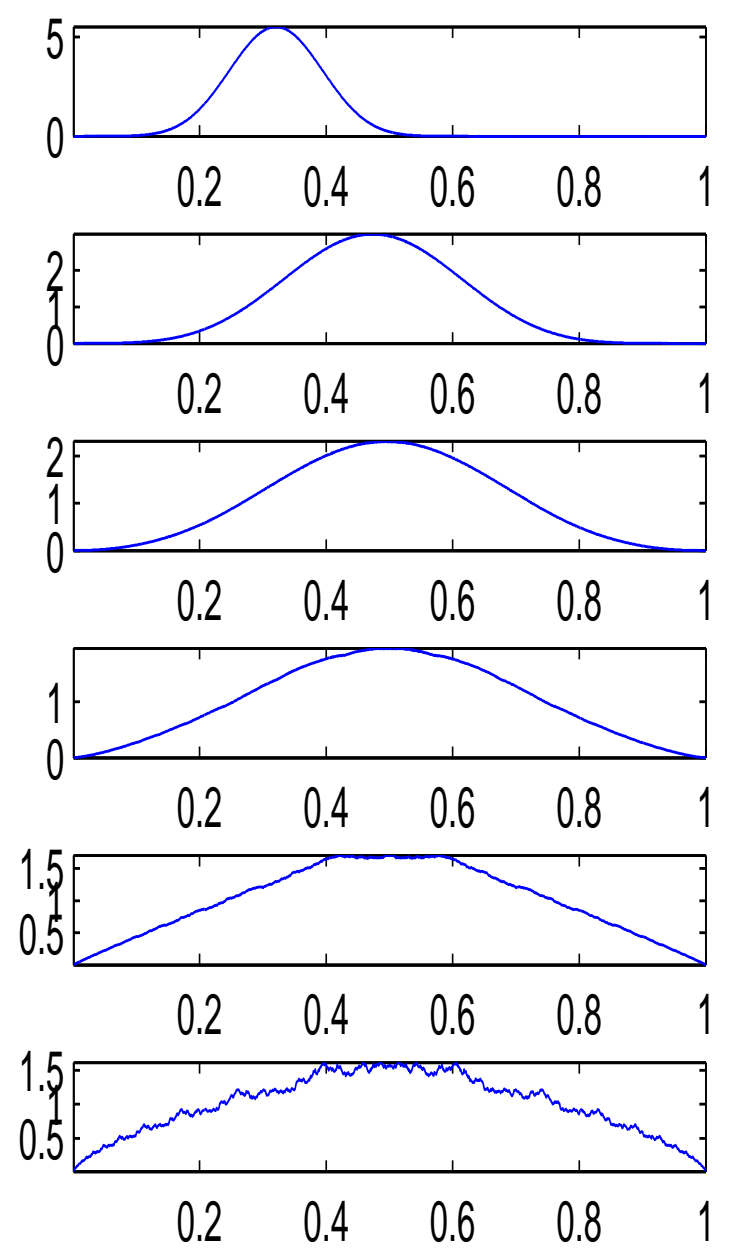

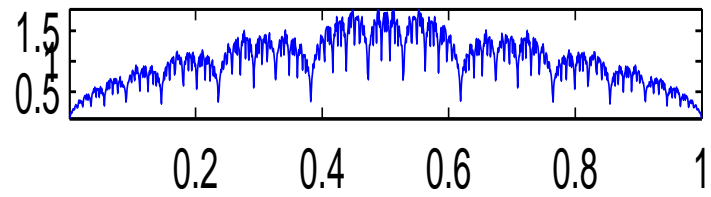

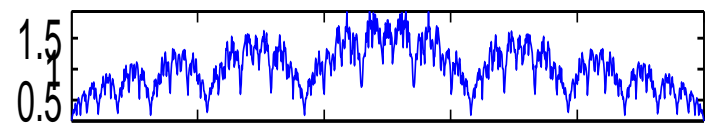

$\begin{array}{lllll}0.2 & 0.4 & 0.6 & 0.8 & 1\end{array}$

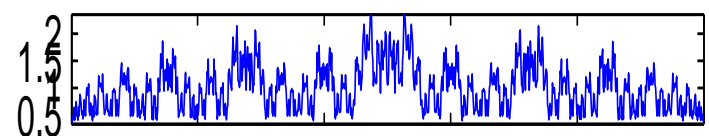

$\begin{array}{lllll}0.2 & 0.4 & 0.6 & 0.8 & 1\end{array}$

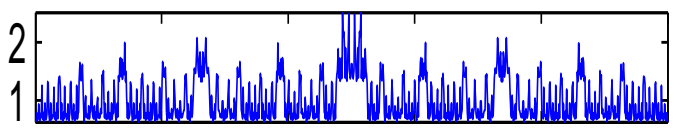

$\begin{array}{lllll}0.2 & 0.4 & 0.6 & 0.8 & 1\end{array}$
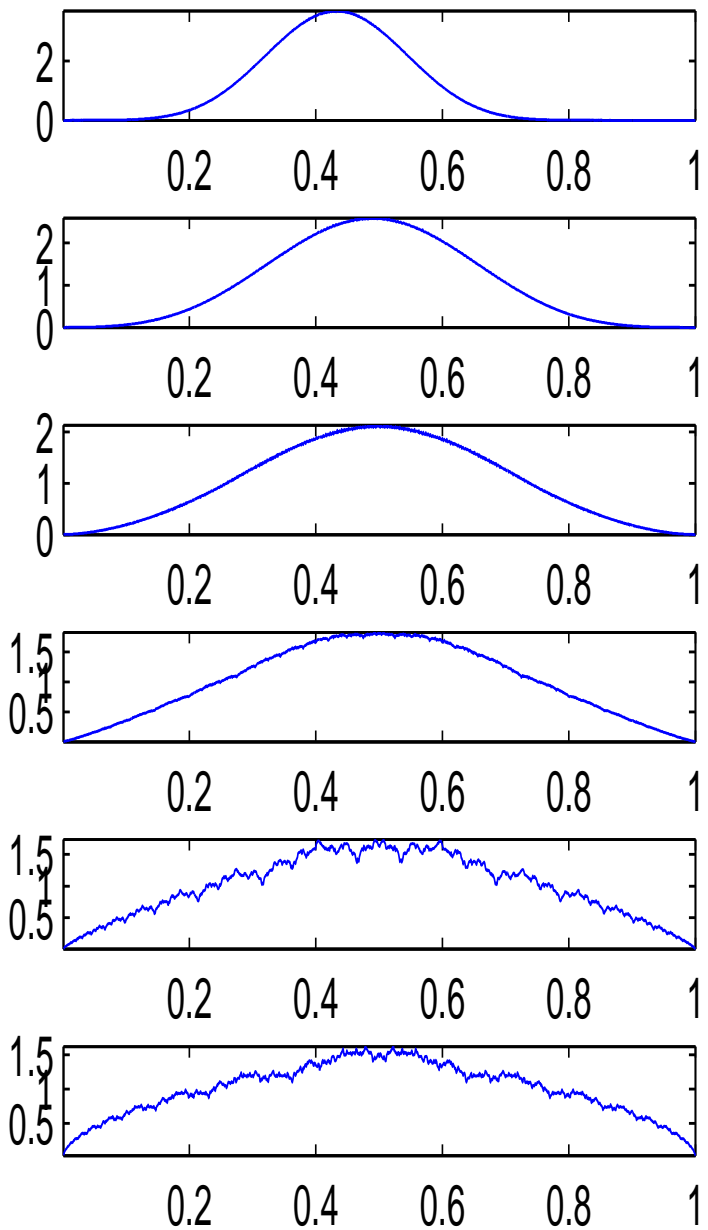

1.5.5 0

$\begin{array}{lllll}0.2 & 0.4 & 0.6 & 0.8 & 1\end{array}$

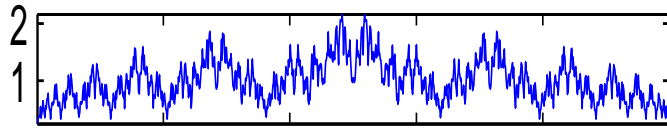

$\begin{array}{lllll}0.2 & 0.4 & 0.6 & 0.8 & 1\end{array}$

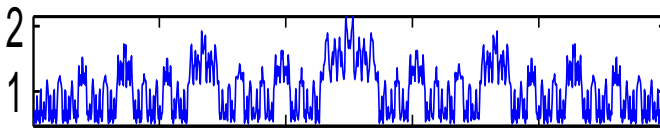
$\begin{array}{lllll}0.2 & 0.4 & 0.6 & 0.8 & 1\end{array}$

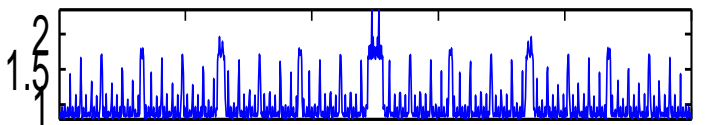

$\begin{array}{lllll}0.2 & 0.4 & 0.6 & 0.8 & 1\end{array}$

Fig 1. Histograms for $F_{\lambda}^{\prime}(t)$ correspond to the following $\lambda^{-1}=1+i / 21, i=1,2, \ldots, 20$ 
- Salem [9-11] proved the converse of the result of Erdos. If $\phi(t)$ does not tend to 0 as $t \rightarrow \infty$, then $\lambda^{-1}$ is necessarily a Pisot number. The reciprocal of Pisot numbers remain today the only known set of $\lambda$ for which $F_{\lambda}(t)$ is singular.

- At the next year Erdös [5] proved a result in opposite direction, namely, there is a $a<1$ such that for almost all $\lambda$ in the interval $a<\lambda<1$ we have $F_{\lambda}(t)$ is absolutely continuous.

- Garsia [6] found new examples of algebraic $\lambda$ with absolutely continuous $F_{\lambda}(t)$.

- Solomyak [12] proved that $F_{\lambda}(t)$ is absolutely continuous with a density in $L^{2}$ for a.e. $0.5<\lambda<1$.

In this paper we study the moments of Bernoulli convolution. They are defined by

$$
M_{n}=\mathrm{E} Y_{\lambda}^{n}=\int_{0}^{1} x^{n} d F_{\lambda}(x) .
$$

The main result of this paper

Theorem 1. Let $M_{n}$ be defined by (4), then the following holds as $n \rightarrow \infty$

$$
M_{n}=n^{\log _{\lambda} 2} 2^{\log _{\lambda}(1-\lambda)+0.5 \log _{\lambda} 2-0.5} e^{\tau\left(-\log _{\lambda} n\right)}\left(1+\mathcal{O}\left(n^{-0.99}\right)\right),
$$

where

$$
\tau(x)=\sum_{k \neq 0} \frac{1}{k} \alpha\left(-\frac{k}{\ln \lambda}\right) e^{2 \pi i k x}
$$

is 1-periodic function,

$$
\alpha(t)=-\frac{1}{2 i \operatorname{sh}\left(\pi^{2} t\right)}(1-\lambda)^{2 \pi i t}\left(1-2^{2 \pi i t}\right) \pi^{-2 \pi i t} 2^{-2 \pi i t} \zeta(2 \pi i t)
$$

and $\zeta(z)$ is the Riemann zeta function.

Remark 1. We emphasize that the periodic function $\tau(x)$ is a constant only in the Wintner's cases $\lambda=2^{-1 / k}, k=1,2, \ldots$.

Moreover, only in these cases $F_{\lambda}(x)=C x^{\alpha}+\mathcal{O}\left(x^{\alpha+\varepsilon}\right)$ for some $\alpha>0, \varepsilon>0$ as $x \rightarrow 0$.

We observe that $|\alpha(t)|$ does not depend on $\lambda,|\alpha( \pm 1 / \ln 2)| \approx 10^{-5}$, and due the fast decrease of $|\alpha(t)|$ (see Figure 2) the fluctuating function $\tau(x)$ stays bounded by $10^{-5}$.

Remark 2. We have the following approximations

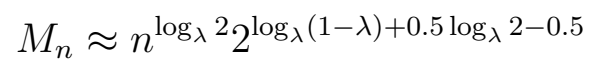

with accuracy $10^{-5}$ and

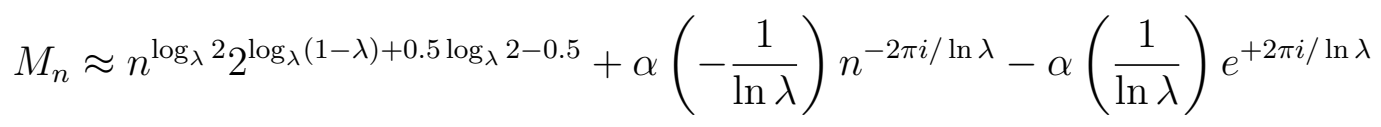

with accuracy $10^{-9}$. 

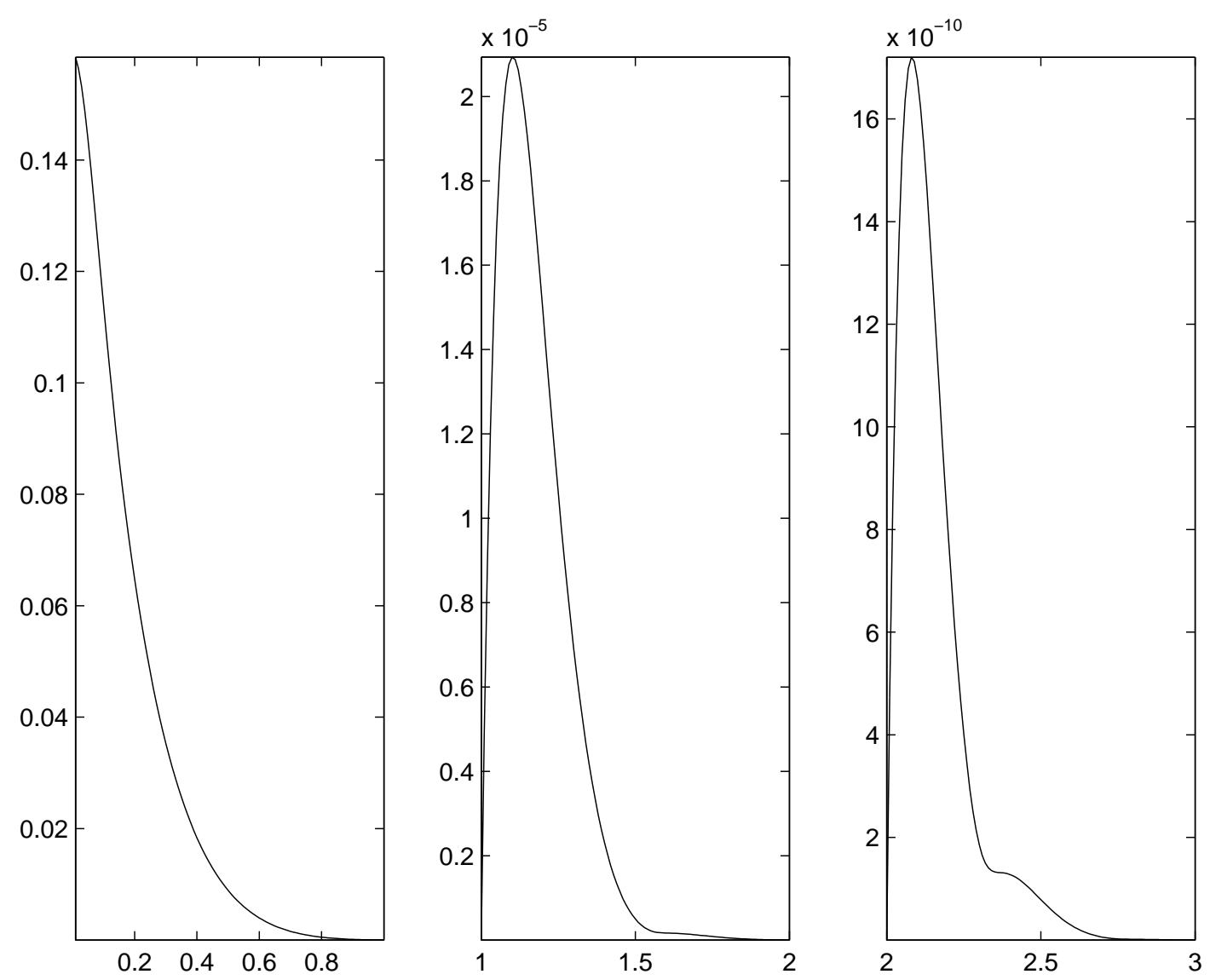

Fig 2. The graphs of $|\alpha(t)|$ for $t \in[0,1], t \in[1,2]$, and $t \in[2,3]$

Proof. Now we give a proof of the theorem using analytic techniques such as poissonization and the Mellin transform

A recursive relation for the moments $M_{n}$ of any function satisfying (1) is the following

$$
\begin{aligned}
& M_{n}=\int_{0}^{1} x^{n} d F_{\lambda}(x)= \\
&=\frac{1}{2} \int_{0}^{1} x^{n} d F_{\lambda}\left(\lambda^{-1} x\right)+\frac{1}{2} \int_{0}^{1} x^{n} d F_{\lambda}\left(\lambda^{-1} x-\lambda^{-1}+1\right)= \\
&=\frac{1}{2} \lambda^{n} \int_{0}^{1} t^{n} d F_{\lambda}(t)+\frac{1}{2} \int_{0}^{1}(\lambda t+1-\lambda)^{n} d F_{\lambda}(t) .
\end{aligned}
$$

Substituting $M_{n}$, we obtain

$$
M_{n}=\frac{1}{2} \lambda^{n} M_{n}+\frac{1}{2} \sum_{k=0}^{n}\left(\begin{array}{l}
n \\
k
\end{array}\right) \lambda^{k}(1-\lambda)^{n-k} M_{k}, \quad M_{0}=1, \quad n=0,1, \ldots
$$

We define the Poisson transform as

$$
M(x)=\sum_{n=0}^{\infty} M_{n} \frac{x^{n}}{n !} e^{-x}
$$


which exists for all complex $x$ since the series converges due to the estimate $M_{m} \leq 1$. Substituting (7) в (8), we get

$$
\begin{array}{r}
M(x)=\begin{array}{r}
\frac{1}{2} \sum_{n=0}^{\infty} \lambda^{n} M_{n} \frac{x^{n}}{n !} e^{-x}+\frac{1}{2} \sum_{n=0}^{\infty} \frac{x^{n}}{n !} e^{-x} \sum_{k=0}^{n}\left(\begin{array}{c}
n \\
k
\end{array}\right) \lambda^{k}(1-\lambda)^{n-k} M_{k}= \\
=\frac{1}{2} M(\lambda x) e^{-(1-\lambda) x}+\frac{1}{2} M(\lambda x) .
\end{array}
\end{array}
$$

Since $M_{0}=1$, we find that

$$
M(x)=\prod_{k=0}^{\infty}\left(\frac{1}{2}+\frac{1}{2} e^{-(1-\lambda) \lambda^{k} x}\right) .
$$

Let

$$
G(x)=\ln M(x)=\sum_{k=0}^{\infty} \ln \left(\frac{1}{2}+\frac{1}{2} e^{-(1-\lambda) \lambda^{k} x}\right) .
$$

The Mellin transform

$$
\tilde{G}(z)=\int_{0}^{\infty} G(x) x^{z-1} d x
$$

is define for

$$
-1<\Re z<0
$$

and satisfies

$$
\tilde{G}(z)=\frac{(1-\lambda)^{-z}}{1-\lambda^{-z}} \int_{0}^{\infty} x^{z-1} \ln \left(\frac{1}{2}+\frac{1}{2} e^{-x}\right) d x,
$$

By integration by parts, we obtain

$$
\tilde{G}(z)=\frac{(1-\lambda)^{-z}}{1-\lambda^{-z}} \frac{1}{z} \int_{0}^{\infty} \frac{x^{z}}{1+e^{x}} d x
$$

Using [15, 3.411.3], we get

$$
\tilde{G}(z)=\frac{(1-\lambda)^{-z}}{1-\lambda^{-z}}\left(1-2^{-z}\right) \Gamma(z) \zeta(z+1)
$$

for $-1<\Re z<0$.

It is known that the Gamma function and the zeta function are both continuable to the complex plane:

- $\Gamma(z)$ has simple poles at the non-positive integers;

- $\zeta(z)$ has only simple pole at $z=1$.

Therefore, the function $\tilde{G}(z)$ continuable to the complex plane.

$\tilde{G}(z)$ has a double pole at $z=0$ and simple poles: 
- at the non-positive integers, from $\Gamma(z)$,

- at $z=z_{k}$, from $\left(1-\lambda^{-z}\right)^{-1}$, where

$$
z_{k}=\frac{2 \pi i k}{\ln \lambda}, \quad k \neq 0
$$

Via the inverse Mellin transform we find that

$$
G(x)=\frac{1}{2 \pi i} \int_{-\sigma-i \infty}^{-\sigma+i \infty} \tilde{G}(z) x^{-z} d z
$$

where $0<\sigma<1$.

In order to find $G(x)$, we apply the residue theorem for the right half-plane $\Re z>-\sigma$.

Now we find residues of the function $\tilde{G}(z) x^{-z}$.

Using the following formula

$$
\operatorname{Res}\left(\frac{P(z)}{z Q(z)}, 0\right)=\frac{P^{\prime}(0)}{Q^{\prime}(0)}-\frac{P(0) Q^{\prime \prime}(0)}{2 Q^{\prime}(0)^{2}},
$$

where

$$
\begin{gathered}
P(z)=\frac{1-2^{-z}}{z} \Gamma(z+1)(1-\lambda)^{-z} x^{-z}(z \zeta(z+1)) \\
Q(z)=1-\lambda^{-z}
\end{gathered}
$$

we obtain

$$
\operatorname{Res}\left(\tilde{G}(z) x^{-z}, 0\right)=\frac{P^{\prime}(0)}{Q^{\prime}(0)}-\frac{P(0) Q^{\prime \prime}(0)}{2 Q^{\prime}(0)^{2}} .
$$

(There $\operatorname{Res}\left(f(z), z_{0}\right)$ denotes the residue of $f(z)$ at the point $z=z_{0}$.)

Substituting $(z \zeta(z+1))_{z=0}=1,(z \zeta(z+1))_{z=0}^{\prime}=-\Gamma^{\prime}(1)[15,9.536]$, we get

$$
\begin{gathered}
P(0)=\ln 2, \quad P^{\prime}(0)=-\ln x \ln 2-\ln (1-\lambda) \ln 2-\frac{1}{2} \ln ^{2} 2, \\
Q^{\prime}(0)=\ln \lambda, \quad Q^{\prime \prime}(0)=-\ln ^{2} \lambda .
\end{gathered}
$$

Therefore,

$$
\operatorname{Res}\left(\tilde{G}(z) x^{-z}, 0\right)=-\frac{\ln 2}{\ln \lambda}\left(\ln x+\ln (1-\lambda)+\frac{1}{2} \ln 2\right)+\frac{1}{2} \ln 2 .
$$

The residue at $z_{k}, k= \pm 1, \pm 2, \ldots$ is

$$
\operatorname{Res}\left(\tilde{G}(z) x^{-z}, z_{k}\right)=\frac{(1-\lambda)^{-z_{k}}}{\ln \lambda}\left(1-2^{-z_{k}}\right) \Gamma\left(z_{k}\right) \zeta\left(z_{k}+1\right) x^{-z_{k}} .
$$

$\Gamma(z)$ decreases exponentially fast along vertical lines while $\zeta(z)$ is only polinomial growth as $\Im z \rightarrow \pm \infty$. Thus, Collorary 1 to Theorem 4 from [3] applies here and we have

$$
\begin{aligned}
G(x)=\frac{\ln 2}{\ln \lambda}(\ln x+ & \left.\ln (1-\lambda)+\frac{1}{2} \ln 2\right)-\frac{1}{2} \ln 2- \\
& -\frac{1}{\ln \lambda} \sum_{k \neq 0}(1-\lambda)^{-z_{k}}\left(1-2^{-z_{k}}\right) \Gamma\left(z_{k}\right) \zeta\left(z_{k}+1\right) x^{-z_{k}}+\mathcal{O}\left(x^{-\gamma}\right),
\end{aligned}
$$


for every $\gamma>0$.

Take $\gamma=\log _{\lambda} 2+2$.

Using (16) and (11), we get

$$
M(x)=x^{\log _{\lambda} 2} e^{\tau\left(\log _{\lambda} x\right)}\left(1+\mathcal{O}\left(x^{-\gamma}\right)\right),
$$

where the function $\tau$ is defined in (5).

In order to find $M_{n}$, we apply Theorem 10.5 from [14]. To apply Theorem 10.5 from [14], we must check that the conditions required in this theorem are actually satisfied. In particular:

there exist $\beta, 0<\theta<\pi / 2$, and $0<\eta<1$ such that the following conditions hold for sufficiently large $|z|, z=x+i y$ :

- for $z \in S_{\theta}$

$$
\left|\frac{1}{2}+\frac{1}{2} e^{-(1-\lambda) z}\right| \lambda^{\beta} \leq 1-\eta
$$

- for $z \notin S_{\theta}$ and some $\alpha<1$

$$
\left|\frac{1}{2}+\frac{1}{2} e^{-(1-\lambda) z}\right| e^{(1-\lambda) x} \leq e^{\alpha(1-\lambda)|z|} ;
$$

where $S_{\theta}=\{z:|\Im z| \leq \theta \Re z\}$.

It can easily be checked that this condition holds for every $\beta>\log _{\lambda} 2$ and we apply Theorem 10.5 from [14] to yeild

$$
M_{n}=M(n)\left(1+\mathcal{O}\left(n^{-0.99}\right)\right) .
$$

for $\beta=\log _{\lambda} 2+0.01$.

The last step consists of simplifying the function $\alpha(t)(6)$. Using (16), we have

$$
\alpha(t)=-\frac{1}{2 \pi i}(1-\lambda)^{2 \pi i t}\left(1-2^{2 \pi i t}\right) \Gamma(-2 \pi i t+1) \zeta(-2 \pi i t+1) .
$$

Applying the functional equation of the Reimann zeta function [15, 9.535.3]

$$
\Gamma(z) \zeta(z) \cos \frac{\pi z}{2}=\pi^{z} 2^{z-1} \zeta(1-z),
$$

we obtain (6).

\section{References}

[1] Bari N.K., Trigonometric Series, Holt, Rinehart and Winston, New York, 1967.

[2] Flajolet P., Sedgewick R., Analytic Combinatorics, Cambridge University Press, 2008.

[3] Flajolet P., Gourdon X., Dumas P., "Mellin transforms and asymptotics: Harmonic sums", Theoretical Computer Science, 144:1-2 (1995), 3-58.

[4] Erdös P., "On a Family of Symmetric Bernoulli Convolutions", American Journal of Mathematics, 61:4 (1995), 974-976. 
[5] Erdös P., "On the Smoothness Properties of a Family of Bernoulli Convolutions", American Journal of Mathematics, 62:1 (1940), 180-186.

[6] Garsia A.M., "Arithmetic Properties of Bernoulli Convolutions", Transactions of the American Mathematical Society, 102:3 (1962), 409-432.

[7] Jessen B., Wintner A., "Distribution Functions and the Riemann Zeta Function", Transactions of the American Mathematical Society, 38:1 (1935), 48-88.

[8] Peres Y., Schlag W., and Solomyak B., "Sixty years of Bernoulli convolutions", Fractals and Stochastics II (C. Bandt, S. Graf and M. Zaehle, eds.), Birkhauser, 2000, 39-65.

[9] Salem R., "Sets of Uniqueness and Sets of Multiplicity", Transactions of the American Mathematical Society, 54:2 (1943), 218-228.

[10] Salem R., "Sets of Uniqueness and Sets of Multiplicity. II", Transactions of the American Mathematical Society, 56:1 (1944), 32-49.

[11] Salem R., "Rectifications to the Papers Sets of Uniqueness and Sets of Multiplicity, I and II", Transactions of the American Mathematical Society, 63:3 (1948), 595-598.

[12] Solomyak B., "On the Random Series $\sum \pm \lambda^{n}$ (an Erdos Problem)", The Annals of Mathematics 2nd Ser., 142:3 (1995), 611-625.

[13] Wintner A., "On Convergent Poisson Convolutions", American Journal of Mathematics, $\mathbf{5 7 : 4}(1935), 827-838$.

[14] Szpankowski W., Average Case Analysis of Algorithms on Sequences, John Wiley \& Sons, New York, 2001.

[15] Gradstein I.S., Ryzhik I.M., Table of integrals, Series, and Products, Academic Press, 1994.

Тимофеев Е.А., "Асимптотика моментов симметричной свертки Бернулли", Моделирование и анализ информачионых систем, 23:2 (2016), 185-194.

DOI: $10.18255 / 1818-1015-2016-2-185-194$

Аннотация. Для каждого $\lambda, 0<\lambda<1$ определим случайную величину (симметричную свертку Бернулли)

$$
Y_{\lambda}=(1-\lambda) \sum_{n=0}^{\infty} \xi_{n} \lambda^{n}
$$

где $\xi_{n}-$ независимые случайные величины с

$$
\mathrm{P}\left\{\xi_{n}=0\right\}=\mathrm{P}\left\{\xi_{n}=1\right\}=\frac{1}{2} .
$$

Основной результат настоящей работы

$$
M_{n}=\mathrm{E} Y_{\lambda}^{n}=n^{\log _{\lambda} 2} 2^{\log _{\lambda}(1-\lambda)+0.5 \log _{\lambda} 2-0.5} e^{\tau\left(-\log _{\lambda} n\right)}\left(1+\mathcal{O}\left(n^{-0.99}\right)\right),
$$

где функция

$$
\tau(x)=\sum_{k \neq 0} \frac{1}{k} \alpha\left(-\frac{k}{\ln \lambda}\right) e^{2 \pi i k x}
$$

является периодической с периодом равным 1 ,

$$
\alpha(t)=-\frac{1}{2 i \operatorname{sh}\left(\pi^{2} t\right)}(1-\lambda)^{2 \pi i t}\left(1-2^{2 \pi i t}\right) \pi^{-2 \pi i t} 2^{-2 \pi i t} \zeta(2 \pi i t),
$$

а $\zeta(z)$ - дзета-функция Римана.

Статья публикуется в авторской редакции. 
Ключевые слова: моменты, самоподобие, свертка Бернулли, сингулярная функция, преобразование Меллина, асимптотика

Об авторах:

Тимофеев Евгений Александрович, orcid.org/0000-0002-0980-2507,

доктор. физ.-мат. наук, профессор кафедры теоретической информатики,

Ярославский государственный университет им. П.Г. Демидова,

ул. Советская, 14, г. Ярославль, 150000 Россия, e-mail: timofeevEA@gmail.com 